\title{
EMPLOYMENT, PRODUCTION AND CONSUMPTION WITH RANDOM UPDATE: NON-EQUILIBRIUM STATIONARY STATE EQUATIONS
}

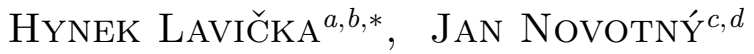 \\ ${ }^{a}$ Czech Technical University in Prague, Faculty of Nuclear Sciences and Physical Engineering, Department of \\ Physics, Břehová 7, CZ-115 19 Praha 1, Czech Republic \\ ${ }^{b}$ Bogolyubov Laboratory of Theoretical Physics, Joint Institute of Nuclear Research, 141980 Dubna, Russia \\ ${ }^{c}$ Centre for Econometric Analysis, Faculty of Finance, Cass Business School, City University London, 106 \\ Bunhill Row, London, EC1Y 8TZ, United Kingdom \\ ${ }^{d}$ CERGE-EI, Charles University, Politickych veznu 936/7, 11000 Prague 1-New Town, Czech Republic \\ * corresponding author: lavicka@fjfi.cvut.cz
}

\begin{abstract}
In this work, we investigate the Model of Employment, Production and Consumption, as introduced in a series of papers by I. Wright [1 3] from the perspective of statistical physics, and we focus on the presence of equilibrium. The model itself belongs to the class of multi-agent computational models, which aim to explain macro-economic behavior using explicit micro-economic interactions. Based on the mean-field approximation, we form the Fokker-Plank equation(s) and then formulate conditions forming the stationary solution, which results in a system of non-linear integral-differential equations. This approximation then allows the presence of non-equilibrium stationary states, where the model is a mixed additive-multiplicative model.
\end{abstract}

KEYWORDS: Multi-agent based model, NESS, equilibrium, Langevin equations.

\section{INTRODUCTION}

In recent years, the emergence of cheap computational power and progress in big data science has resulted in the appearance of multi-agent computational models in the economic and social sciences, see [4]. These models aim to model macroscopic properties of the socioeconomic system, using explicit modeling of microscopic interactions. Such interactions are mimicked explicitly as a large-scale experiment, where thousands of agents are equipped with a set of rules and their mutual interaction is explicitly modeled using Monte Carlo procedures, see [5] for a number of explicit examples.

Large scale multi-agent computational models are, by their nature, black-box computational models, with no direct relation between the variables of interest. This is the main critique in comparison with main stream economic tools, which rather aim to find an explicit and analytically tractable link between the variables. By contrasts, multi-agent computational models have to be perceived as complex systems, see [5- 7], and this requires a specific set of tools. In particular, there is a question about the existence of "equilibrium", which is a key component of many economic models.

The contribution of our paper is two-fold. First, the paper aims to establish a serious discussion about investigating the presence and the type of equilibrium in complex socioeconomic systems. To the best of the knowledge of the authors' such a discussion is missing in the literature, and thus there is no bridge between standard economic models and complex multi-agent based models. Second, we illustrate our point with the model of employment, production and consumption employed in [1,3, 8, 13, which mimics the economic activity in society. For this model, we utilize the mean-field approximation, which illustrates the presence of non-equilibrium stationary states and contradicts the conventional economic intuition. An answer to the question about the qualitative properties of the states that the economy reaches precedes any attempts to find quantitative solutions.

The paper is organized as follows. In Section 2, we define the model of employment, production and consumption. In Section 3, we employ the mean-field approximation and the qualitative properties of the solution. Section 4 provides conclusions.

\section{Definition OF THE MODEL}

The model of Employment, Production and Consumption (EPC, henceforth) was originally proposed in a series of papers by Ian Wright [1 3]. The model is based on the social stratification of society according to an individual's holding and utilization of capital, as a means of production and wealth. The model assumes and explicitly models two distinct types of agents: citizens and companies. The number of citizens is held constant 
in the model, while the number of companies varies dynamically and endogenously over time. The model itself represents a grand-canonical ensemble with respect to the agents in the economy as the number of companies varies endogenously over time.

Economic activity in the economy is undertaken by companies, which are the only agents who can compete on the market. Companies, however, are endogenously created by citizens and cannot operate without them. Thus, every citizen in the economy is characterized by her status with respect to companies: she can be at any time either unemployed, employed or the owner of a company. Both citizens and companies are characterized by their holding of capital, serving the role of wealth. The model itself is characterized by the properties of the individual agents and the macroscopic properties of the system, e.g. the rate of employment of agents or the distribution of capital among different groups of citizens is the result of individual interactions among agents. It is worth noting that the model itself can be highly non-linear with respect the parameters (exogenous conditions) can produce a non-linear response, as has been illustrated by [8].

The definition of the model is in the form of Langevin equations with discrete time steps, using multi-step procedure driving the dynamics. The economic activity mimics the procedure where citizens demand goods to consume, which is in turn satisfied by the economic activity of companies, which supply the goods to consume. The concept of goods is implicit, though, and all the economic transactions are proxied in terms of capital.

Each micro-state of the system is described by the parameters of each of $N$ citizens and the volume of demanded goods $D$. The state of the corporate sector can be deduced from the knowledge of citizens. In particular, a citizen $i$ carries three variables $\left\{m_{i}, e_{i}, \eta_{i}\right\}$, where $m_{i}$ stands for the total amount of money owned, $e_{i}$ specifies one of the three states with respect to the corporate sector, and, finally, $\eta_{i}$ is a wage expectation. The state of the system at time $t$ is thus described by $S_{t} \equiv\left\{\left\{m_{i}, e_{i}, \eta_{i}\right\}_{i \in\{1, \ldots, N\}}, D\right\}$. In particular, if $e_{i}$ points to the same agent, it denotes an owner of a company. If it points toward another agent, she is an employee of the other agent. And if it is empty, she is unemployed.

The commercial cycle which propels the dynamics consists of four turns:

- Hiring turn;

- Demand turn;

- Revenue turn;

- Wage turn;

where during each turn, a certain part of the system is updated. Passing through all sub-steps evolves the system one step ahead in time. As the system passes intermediate states, we equip it with superscripts $H, D$ and $R$, indicating that it is a result of the Hiring turn, the Demand turn and the Revenue turn, respectively. The end of the Wage turn coincides with the end of the entire step.

This model was originally defined by Wright in [2], following his research in [1, 3, 14, 15]. In this paper, we generalize his definitions using a general notation of the functions $f_{A}(w), f_{D}(w)$, and $f_{R}(D)$ of individual probability densities employed during the consecutive turns, which depends on one parameter and we also make changes such that the model is a fully random updated mode $\sqrt{1}$. In the definitions below, we assume that the random variables are mutually uncorrelated, undergoing a certain well-defined probability distribution, if not specified otherwise.

\subsection{THE HIRING TURN}

During the first turn, an unemployed citizen can either become employed or can set up her own venture and become an employer. Each unemployed citizen evaluates her attractiveness towards each of the existing employers and other unemployed citizens (denoted as $\mathcal{H}$ ). Attractiveness is a function of the other agent's wealth. Then, each unemployed citizen chooses a new employer indexed by $h$ with probability $\mathbb{P}\left(w_{h}\right)=\frac{f_{A}\left(w_{h}\right)}{\sum_{i \in \mathcal{H}} f_{A}\left(w_{i}\right)}$, where $\mathbb{P}\left(w_{h}\right)$ serves as a weight to choose a potential employer $h$. Then, the unemployed person decides whether she will turn her initial inclination into a contract. The decision is based on the following rule: she draws a random number from $\eta_{c}^{H} \sim U\left(\eta_{c}, 2 \eta_{c}\right)$, where $\eta_{c}$ are the agent's wage expectations, and if $\eta_{c}^{H} \leq w_{h}$ then she either joins the existing company of agent $h$ or initiates the creation of a new venture by agent $h$ (the creation of a company is induced by the demand for employment). Employment increases the future wage expectations, as $\eta_{c} \leftarrow \eta_{c}^{H}$. Otherwise, she remains unemployed and her wage expectations for the next turn decrease as $\eta_{c} \leftarrow U\left(0, \eta_{c}\right)$.

\subsection{THE DEMAND TURN}

In the next turn, citizens consume goods available on the market and thus create demand for them. Each citizen $c$ spends a share of her endowment $d_{c}^{D}$ on goods. The amount to spend is expressed as $d_{c}^{D} \sim f_{D}\left(w_{c}\right)$, where

\footnotetext{
${ }^{1}$ Article 2 proposes a model where a wage is paid to all agents at the same time. This, however, represents a multi-body interaction, which introduces an unnecessary degree of complexity. We replace this step by a sequential two-body interaction, which means that the wage is paid consequently, agent by agent.
} 
$f_{D}(w)$ is some probability distribution and is a function of the agent's wealth. After this spending on goods, she owns $w_{c}^{D}=w_{c}-d_{c}^{D}$ and the pool of demanded goods increases as $D^{D}=D+d_{c}^{D}$.

\subsection{THE REVENUE TURN}

Then, the companies aim to exploit the pool of demanded goods and compete for citizens. Each citizen $c$ from the pool of companies and company owners works for her company and supplies a share of demanded goods $d_{c}^{R}$. The supplied amount of goods is expressed as $d_{c}^{R} \sim f_{R}\left(D^{D}\right)$, where $f_{R}(D)$ is a probability distribution. Consequently, the demand is decreased as $D^{R}=D^{D}-d_{c}^{R}$. For an employee $c, d_{c}^{R}$ is sent to the company owner's budget as $w_{e_{c}}^{R}=w_{e_{c}}^{H}+d_{c}^{R}$, where $e_{c}$ is a pointer to the appropriate employer. For an employer, on the other hand, the rule reads as $w_{c}^{R}=w_{c}^{H}+d_{c}^{R}$.

\subsection{The WAGE TURN}

Finally, each employee is paid for her work for the company. Employee $c$ obtains her wage $\eta_{c}$, which is based on her initial wage expectations, which were agreed during the employment contract. If employer $e_{c}$ has enough resources to provide this wage, the wage is paid and thus $w_{c}=w_{c}^{D}+\eta_{c}$ and $w_{e_{c}}=w_{e_{c}}^{D}-\eta_{c}$. If $w_{e_{c}}<\eta_{c}$, on the other hand, the employer does not have enough resources, the employee receives what remains as $w_{c}=w_{c}^{D}+w_{e_{c}}$ and then becomes unemployed. If the employer loses all her employees, she becomes unemployed as well.

\section{Derivation of the Stationary state EQUATIONS}

The model introduced in the preceding section is a Markov process that evolves micro-states of the system described by $4 \cdot N+1$ variables in total, captured by $S_{t} \equiv\left\{\left\{m_{i}, e_{i}, \eta_{i}\right\}_{i \in\{1, \ldots, N\}}, D\right\}$ at time $t$. The time coordinate can point to any turn at each step; however, we will mainly refer to time $t$ after all four turns have been completed.

Each time step state of the system evolves following the transition probabilities $\mathbb{P}\left(S_{t} \mid S_{t}^{\prime}\right)$, which move the system among the micro-states, using the rule

$$
\mathbb{P}\left(S_{t+\triangle t} \mid S_{0}\right)=\mathbb{P}\left(S_{t+\triangle t} \mid S_{t}^{\prime}\right) \cdot \mathbb{P}\left(S_{t}^{\prime} \mid S_{0}\right)
$$

The solution $\mathbb{P}\left(S_{t} \mid S_{0}\right)$ of the system with the initial micro-state $S_{0}$ can be very complex, and even impossible to express with a system of analytically solvable equations. Therefore, we rather focus on the ground stationary state $\mathbb{P}(S) \equiv \lim _{t \rightarrow+\infty} \mathbb{P}\left(S_{t} \mid S_{0}\right)$ which is a solution to the equation

$$
\mathbb{P}(S)=\mathbb{P}\left(S \mid S^{\prime}\right) \mathbb{P}\left(S^{\prime}\right)
$$

Thus the stationary state is an eigenvector of eigenvalue 1 of the transition probabilities if the equations are linear. Our model, in contrast to such classical models as the Ising model, ASEP and Brownian motion (see, for example 16, and 17) is described by a set of different sub-transition probabilities following each described turn, thus composing the total transition probability as follows

$$
\mathbb{P}\left(S \mid S^{\prime}\right)=\mathbb{P}^{W}\left(S \mid S^{I I I}\right) \mathbb{P}^{R}\left(S^{I I I} \mid S^{I I}\right) \mathbb{P}^{D}\left(S^{I I} \mid S^{I}\right) \mathbb{P}^{H}\left(S^{I} \mid S^{\prime}\right),
$$

where $\mathbb{P}^{H}\left(S^{I} \mid S^{\prime}\right), \mathbb{P}^{D}\left(S^{I I} \mid S^{I}\right), \mathbb{P}^{R}\left(S^{I I I} \mid S^{I I}\right)$ and $\mathbb{P}^{R}\left(S^{I I I} \mid S^{I I}\right)$ stands for the Hiring, Demand, Revenue and Wage turns, respectively.

We simplify the problem (1) using a set of ansatzes, which we impose on the stationary solution. Each particular ansatz decreases the complexity of the problem, assuming the independence of certain variables.

First, we assume that

$$
\mathbb{P}(S) \equiv \mathbb{P}\left(\left\{w_{i}, e_{i}, \eta_{i}\right\}, D\right) \simeq \mathbb{P}\left(\left\{w_{i}, e_{i}, \eta_{i}\right\}\right) \mathbb{P}_{D}(D),
$$

i.e., independence of the level of demand from the particular state of the society.

Second, we assume the independence of wage expectations from the state of the entire system, or,

$$
\mathbb{P}\left(\left\{w_{i}, e_{i}, \eta_{i}\right\}\right) \simeq \mathbb{P}\left(\left\{w_{i}, e_{i}\right\}\right) \mathbb{P}_{\eta}(\eta)
$$

Third, we employ the mean-field approximation of employment contracts, and thus assume

$$
\mathbb{P}\left(\left\{w_{i}, e_{i}\right\}\right) \simeq \mathbb{P}\left(X, w_{X}\right)
$$

Then, the stationary state of the model is identified using the above set of ansatzes, in the form of

$$
\mathbb{P}(S) \equiv \mathbb{P}\left(\left\{w_{i}, e_{i}, \eta_{i}\right\}, D\right) \simeq \mathbb{P}_{D}(D) \mathbb{P}_{\eta}(\eta) \mathbb{P}\left(X, w_{X}\right)
$$


where the entire system is described by variables $X$ and $w_{X}$, where $X \in\{U, E, C\}$ substitutes the status with respect to companies and $w_{X}$ shows the accumulated wealth. The state of economy is than described by 3 variables $D, \eta$ and $m$, which describe aggregated demand, wage expectation, and income, respectively. We assume (3) for each variable in each turn of the system in decomposition (2).

During each of the turns of the economic activity, the system changes, and we thus equip in each turn the variables with superscript $(H, D$, and $R)$ denoting the particular turn. We first define the function

$$
W(w)=\int_{w}^{+\infty} f_{A}\left(w^{\prime}\right)\left(\mathbb{P}\left(U, w^{\prime}\right)+\mathbb{P}\left(C, w^{\prime}\right)\right) \mathrm{d} w^{\prime},
$$

and a constant

$$
C_{1}=\frac{\int_{0}^{+\infty} W(\eta) \mathbb{P}_{\eta}(\eta) \mathrm{d} \eta}{W(0)}
$$

\subsection{The Hiring Turn}

During the hiring turn, the variables of the system evolve as follows

$$
\begin{aligned}
\mathbb{P}^{H}\left(E, w_{E}\right) & =\mathbb{P}\left(E, w_{E}\right)+\mathbb{P}\left(U, w_{E}\right) C_{1} \\
\mathbb{P}^{H}\left(C, w_{C}\right) & =\mathbb{P}\left(C, w_{C}\right)+\mathbb{P}\left(U, w_{C}\right) \frac{f_{A}\left(w_{C}\right) \int_{0}^{w_{C}} \mathbb{P}_{\eta}(\eta) \mathrm{d} \eta}{W(0)} \\
\mathbb{P}^{H}\left(U, w_{U}\right) & =\mathbb{P}\left(U, w_{U}\right)-\mathbb{P}\left(U, w_{U}\right) C_{1}-\mathbb{P}\left(U, w_{U}\right) \frac{f_{A}\left(w_{U}\right) \int_{0}^{w_{U}} \mathbb{P}_{\eta}(\eta) \mathrm{d} \eta}{W(0)}, \\
\mathbb{P}^{H}(\eta) & =C_{1} \int_{\frac{\eta}{2}}^{\eta} \frac{\mathbb{P}_{\eta}\left(\eta^{\prime}\right)}{\eta^{\prime}} \mathrm{d} \eta^{\prime}+\left(1-C_{1}\right) \int_{\eta}^{+\infty} \frac{\mathbb{P}_{\eta}\left(\eta^{\prime}\right)}{\eta^{\prime}} \mathrm{d} \eta^{\prime}
\end{aligned}
$$

\subsection{The DEMAND TURN}

Consequently, during the demand turn, the following update is performed:

$$
\begin{aligned}
\mathbb{P}^{D}\left(X, w_{X}\right) & =\int_{0}^{+\infty} \mathbb{P}^{H}\left(X, w_{X}+d\right) f_{D}\left(d, w_{X}+d\right) \mathrm{d} d \\
\mathbb{P}_{D}^{D}(D, t) & =\int_{0}^{D} \iiint_{\mathcal{R}_{+}^{3}} \prod_{X \in\{U, E, C\}} f_{D}\left(w_{X}^{D}, w_{X}\right) \mathbb{P}^{H}\left(X, w_{X}\right) \delta\left(\sum_{X \in\{U, E, C\}} w_{X}^{D}-d\right) \mathrm{d} w_{X} \mathbb{P}_{D}(D-d, t) \mathrm{d} d
\end{aligned}
$$

where the delta function $\delta\left(x-x_{0}\right)$ in $(9)$ effectively decreases the dimension of integration by 1 , and $\mathcal{R}_{+}$is a set of positive real numbers.

\subsection{The REVENUE TURN}

During the revenue turn, the system changes as

$$
\begin{aligned}
\mathbb{P}^{R}\left(C, w_{C}\right) & =C_{2} \int_{0}^{w_{C}} \mathbb{P}^{D}\left(C, w^{\prime}-D^{\prime}\right) \int_{0}^{+\infty} \mathbb{P}_{D}^{D}\left(D^{\prime}\right) f_{R}\left(m^{\prime}, D^{\prime}\right) \mathrm{d} D^{\prime} \mathrm{d} w^{\prime}+\left(1-C_{2}\right) \mathbb{P}^{D}\left(C, w_{C}\right), \\
\mathbb{P}_{D}^{R}(D) & =C_{2} \int_{0}^{+\infty} \mathbb{P}_{D}^{D}\left(D^{\prime}+D\right) f_{R}\left(D^{\prime}, D^{\prime}+D\right) \mathrm{d} D^{\prime}+\left(1-C_{2}\right) \mathbb{P}_{D}^{D}(D),
\end{aligned}
$$

where $C_{2}=\int_{0}^{+\infty}(\mathbb{P}(E, w)+\mathbb{P}(C, w)) \mathrm{d} w$. 


\subsection{THE WAGE TURN}

Finally, the wage turn evolves according to equations

$$
\begin{aligned}
& \mathbb{P}\left(E, w_{E}\right)=-\mathbb{P}^{D}\left(E, w_{E}\right) \int_{0}^{+\infty} \int_{0}^{\eta} \mathbb{P}_{\eta}^{H}(\eta) \mathbb{P}^{R}\left(C, w^{\prime}\right) \mathrm{d} w^{\prime} \mathrm{d} \eta+\int_{0}^{w_{E}} \mathbb{P}\left(E, w-w^{\prime}\right) \int_{0}^{+\infty} \mathbb{P}_{\eta}^{H}\left(w^{\prime}\right) \mathbb{P}^{R}\left(C, w^{\prime \prime}+w^{\prime}\right) \mathrm{d} w^{\prime \prime} \mathrm{d} w^{\prime}, \\
& \mathbb{P}\left(C, w_{C}\right)= \mathbb{P}^{D}\left(C, w_{C}\right)\left(1-\frac{\int_{0}^{+\infty} \mathbb{P}\left(C, w^{\prime}\right) \mathrm{d} w^{\prime}}{\int_{0}^{+\infty} \mathbb{P}\left(E, w^{\prime}\right) \mathrm{d} w^{\prime}} \int_{0}^{+\infty} \int_{0}^{\eta} \mathbb{P}_{\eta}^{H}(\eta) \mathbb{P}^{R}\left(C, w^{\prime}\right) \mathrm{d} w^{\prime} \mathrm{d} \eta\right) \\
& \mathbb{P}\left(U, w_{U}\right)= \mathbb{P}^{D}\left(U, w_{U}\right)+\mathbb{P}^{D}\left(E, w_{E}\right) \int_{0}^{+\infty} \int_{0}^{+\infty} \mathbb{P}_{\eta}^{H}(\eta) \mathbb{P}^{R}\left(C, w^{\prime}\right) \mathrm{d} w^{\prime} \mathrm{d} \eta \\
&+\mathbb{P}^{D}\left(C, w_{U}\right) \frac{\int_{0}^{+\infty} \mathbb{P}\left(C, w^{\prime}\right) \mathrm{d} w^{\prime}}{\int_{0}^{+\infty} \mathbb{P}\left(E, w^{\prime}\right) \mathrm{d} w^{\prime}} \int_{0}^{+\infty} \int_{0}^{\eta} \mathbb{P}_{\eta}^{H}(\eta) \mathbb{P}^{R}\left(C, w^{\prime}\right) \mathrm{d} w^{\prime} \mathrm{d} \eta \\
& \mathbb{P}_{\eta}(\eta)= \int_{0}^{+\infty} \mathbb{P}_{\eta}^{H}\left(\eta+w^{\prime}\right) \frac{\mathbb{P}^{D}\left(C, w^{\prime}\right)}{\int_{0}^{+\infty} \mathbb{P}^{D}\left(C, w^{\prime \prime}\right) \mathrm{d} w^{\prime \prime}} \mathrm{d} w^{\prime}+\mathbb{P}_{\eta}^{H}(\eta)\left(1-\frac{\int_{0}^{\eta} \mathbb{P}^{D}\left(C, w^{\prime}\right) \mathrm{d} w^{\prime}}{\int_{0}^{+\infty} \mathbb{P}^{D}\left(C, w^{\prime \prime}\right) \mathrm{d} w^{\prime \prime}}\right),
\end{aligned}
$$

where we have omitted any superscript as the result coincides with the final stage of the economy.

\subsection{Discussion ON PROPERTIES OF THE SOLUTION}

The system of equations (4)- 15 describes the ground stationary state of a non-equilibrium macro-economic model (the transition probabilities for macro-state variables) based on the micro-economic characteristics of individual agents. The model is as a Fokker-Planck equation using the mean-field approximation. The equations constitute a closed non-linear system. The system of equations is formulated in general and it may then be simplified using a certain class of microscopic characteristics captured by $f_{A}(w), f_{R}(D)$ and $f_{D}(d, w)$. In the case of Wright's EPC model, see [2], the mean-field approximation can be obtained using $f_{A}(w)=w$, $f_{R}(m, D)=\frac{1}{D}$ and $f_{D}(d, w)=\frac{1}{w}$. Let us note that the system of equations $44-(15)$ is the simplest formulation that exhibits the micro-structure of particular economic clusters of citizens and their mutual interactions. Generally, more simplifications can be made at the expense of reducing the accuracy of the model and its connection to microscopic properties.

To calculate the "excited stationary states" of the system, we have to modify the system of equations (11)-(15) with a prefactor constant $\lambda$ on the left hand side of equations (4)-(7). The prefactor must be determined prior to solution of the system. Excited states are eigenvectors (in linear case) of a composite operator $\left\{\mathbb{P}^{(n)}\left(X, w_{X}\right), \mathbb{P}_{D}^{(n)}(D), \mathbb{P}_{\eta}^{(n)}(\eta)\right\}$ associated with eigenvalues $\lambda_{n}$ and the system of equations 11 - 15 is a matrix equation. Eigenvalues are roots of the characteristic polynomial $\mathcal{P}(\lambda)=0$ associated to such a matrix system.

Uniqueness of the stationary state is an open question but non-uniqueness would allow the existence of different phases of the system with systematically different states of the economy, where a change of the stationary state would require an action of the exogenous field - in the economic case, a policy action by government or by a policy-making body.

Finally, in the case of non-equilibrium stationary states, there exists a non-zero expected macro-scopic current (currents), while equilibrium states do not allow for any non-zero macroscopic currents. In the context of our model, such a current can be represented by the flow of capital or by the structure of the society with respect to the corporate sector. To illustrate the existence of such currents, let us consider the Hiring turn, where the number of employees or the size of the corporate sector increases due to random fluctuations in the model. Therefore, there is a directed flow of agents into employment or corporate ownership, or, equivalently, an outflow of agents from unemployment. On the other hand, during the Wage turn, we observe a similar flow but with a different sign.

However, on the scale of the entire commercial cycle, we observe an equilibrium. This follows from the fact that there are no exogenous driving forces, active boundaries or external reservoir(s). In the case of the economic system, these exogenous factors can be illustrated by supporting particular industry or subsidizing newly set 
up ventures (for driving forces), imposing a minimum wage and a pension system (for active boundaries), and trading with foreign economies with an active international trade balance sheet (for external reservoirs). Therefore, the model presented here is clearly of the non-equilibrium stationary state type.

\section{Conclusions And discussion}

In this paper, we have discussed the characteristics of the model of employment, production and consumption presented in [2], which mimics the economic activity in a society using the multi-agent computational model. We have provided a novel derivation using mean-field theory, and we have obtained a set of non-equilibrium ground stationary state equations for the model initially described by Fokker-Planck equations. In contrast to simple models like one-step Brownian motion, see [17, various versions of the SSEP and ASEP models, see [16] and references therein, or various examples of kinetics, see [18, we have derived a set of equations that drives the mean-field approximation and are of non-linear integral-differential type.

The set of identified dynamic equations is too complex, and a full analytic treatment of it is not feasible. General complexity lies in the conditional interactions which produce non-linear terms, where another source of complexity lies in combination of multiplicative and additive processes during the commercial cycle, where the wage expectations undergo a multiplicative process - the mean of this process is governed by extreme events - while the rest undergoes an additive process - with means being governed by most likely events, as was elaborated in [19]. Our solution can be thus explained from the perspective of [20, 21], while the non-linear multi-dimensional case present in our model is a novelty.

Our paper provides an initial discussion on the analytic treatment of the EPC multi-agent computational model. The results suggest several extensions. Firstly, the numerical solution to the approximate analytic description can be compared with the direct Monte Carlo simulation of the system, and thus the loss we suffer in the mean-field approximation can be evaluated. Second, the solutions suggest that sudden changes of currents related to the stationary state due to a change in the exogenous parameters are possible. However Wright's original model does not have any external parameter, in contrast to the parallel formulation of the EPC model, see $[8$. Thus, the formulation of 8 is a natural extension of the study presented here. Finally, from the thermodynamic perspective, the model resembles the Mimkes model of a productive economy, based on the Carnot cycle with two reservoirs, see [13]. This further stresses the possible existence of a non-equilibrium stationary state of the economy, which contradicts the recent economic paradigm based on equilibrium concepts. All these extensions are left for future research.

In conclusion, our analyzed model is in equilibrium on the large scale; however, it is composed of nonequilibrium intermediate states. Multi-agent computational models are, in turn, very likely to exhibit such non-equilibrium behavior in the intermediate states. The dynamics of multi-agent computational models is therefore more general than compared dynamics of models based on equilbrium assumptions in every intermediate step, and thus allows more complex socio-economic behavior be mimicked.

\section{ACKNOWLEDGEMENTS}

We would like to express our thanks for material and financial support from GACR grant No. P402/12/2255, and from grant RVO68407700 of the Czech Ministry of Education in support of the development of the Sunrise cluster, where some of the calculations were executed. JN acknowledges funding from the European Community's Seventh Framework Program FP7-PEOPLE-2011-IEF under grant agreement number PIEF-GA-2011-302098 (Price Jump Dynamics).

\section{REFERENCES}

[1] I. Wright. The Social Structure of Capitalism. Physica A 346:589-620, 2005.

[2] I. Wright. Implicit Microfoundations for Macroeconomics. Economics: The Open-Access, Open-Assessment E-Journal 3(2009-19), 2009.

[3] I. Wright. The emergence of the law of value in a dynamic simple commodity economy. Review of Political Economy 20:367-391, 2008.

[4] D. Helbing. Economics 2.0: The natural step towards a self-regulating, participatory market society, 2013. Preprint, arXiv:1305.4078.

[5] L. Tesfatsion, K. L. Judd. Handbook of computational economics, Vol. 2 : agent-based computational economics (1st ed.). Amsterdam, NL: Elsevier, 2006.

[6] H. Lavička. Simulations of Agents on Social Network. LAP Lambert Academic Publishing, 2010.

[7] P. W. Anderson, K. J. Arrow, D. Pines. The Economy as an evolving Complex System,. Addison-Wesley, Reading, MA., 1988.

[8] H. Lavička, L. Lin, J. Novotný. Employment, Production and Consumption Model: Patterns of Phase Transition. Physica A 389:1708-1720, 2010. 
[9] H. Dawid, S. Gemkow, P. Harting, et al. The Eurace@Unibi Model: An Agent-based Macroeconomic Model for Economic Policy Analysis. Working paper Universität Bielefeld 2012.

[10] G. Dosi, G. Fagiolo, A. Roventini. Schumpeter meeting Keynes: A policy-friendly model of endogenous growth and business cycles. Journal of Economic Dynamics \& Control 24:1748-1767, 2010.

[11] T. Assenza, G. D. Delli. E Pluribus Unum: Macroeconomic Modelling for Multi-agent Economies. Gredeg working papers, GREDEG CNRS, University of Nice-Sophia Antipolis, 2012.

[12] L. Lin. Some extensions to the social architecture model. In J. Wells, E. Sheppard, I. Wright (eds.), Proceedings of Probabilistic Political Economy: Law of Chaos in the 21st Century. Kingston University, 2008.

[13] J. Mimkes. The Complex Networks of Economic Interactions, vol. 567, chap. Concepts of Thermodynamics in Economic Growth, pp. 139-152. Springer Berlin Heidelberg, 2006.

[14] I. Wright. A conjecture on the distribution of firm profit. Economía: Teoría y Práctica 20, 2004.

[15] I. Wright. The Duration of Recessions Follows an Exponential not a Power Law. Physica A 345:608-610, 2004.

[16] B. Derrida. Non-equilibrium steady states: fluctuations and large deviations of the density and of the current. $J$ Stat Mech P07023, 2007.

[17] A. Einstein. Über die von der molekularkinetischen Theorie der Wärme geforderte Bewegung von in ruhenden Flüssigkeiten suspendierten Teilchen. Annalen der Physik 17:549-560, 1905.

[18] P. Krapivsky, S. Redner, E. Ben-Naim. A kinetic View of Statistical Physics. Cambridge University Press, 2010.

[19] S. Redner. Random multiplicative processes: An Elementary Tutorial. Am Phys J 58(3):267-273, 1990.

[20] D. Sornette, R. Cont. Convergent multiplicative processes repelled from zero: Power law and truncated power laws. J Phys I France 7:431-444, 1997.

[21] D. Sornette. Multiplicative processes and power laws. Phys Rev E 57:4811-4813, 1998. 